\title{
Vacinação em tempos de fake news: um olhar sobre a literatura
}

\author{
Vaccination in fake news times: a look at literature \\ Vacunación en falsas noticias: una mirada a la literatura
}

\begin{abstract}
RESUMO
Objetivo: compreender os aspectos teórico-conceituais relacionados à vacinação e as implicações das Fake news. Método: Trata-se de uma revisão narrativa da literatura, realizou-se as buscas nas bases Scientific Electronic Library Online (SciELO), Literatura Latino-americana e do Caribe em Ciências da Saúde (Lilacs) e Google Scholar, sendo selecionados 11 artigos para análise. A busca se deu nos meses abril e maio de 2020. Resultados: A adesão à vacinação é observada como uma importante estratégia que busca ampliar a cobertura vacinal. Na atualidade, é possível afirmar que as mídias sociais são os principais meios de propagação das Fake News sobre vacinação, e a partir disto pode interferir na diminuição da cobertura vacinal. Conclusão: 0 estudo acena para a necessidade da ressignificação das práticas de vacinação por parte dos profissionais e usuários, com vistas a disseminar informações verídicas e consolidar a vacinação como medida preventiva de doenças.
\end{abstract}

DESCRITORES: Vacinação; Acesso à informação; Programas de imunização.

\section{ABSTRACT}

Objective: to understand the theoretical and conceptual aspects related to vaccination and the implications of Fake news. Method: It is a narrative review of the literature, searches were carried out at the bases Scientific Electronic Library Online (SciELO), Latin American and Caribbean Literature on Health Sciences (Lilacs) and Google Scholar, with 11 articles selected for analysis. The search took place in April and May 2020. Results: Adherence to vaccination is seen as an important strategy that seeks to expand vaccination coverage. Nowadays, it is possible to affirm that social media are the main means of spreading Fake News about vaccination, and from this it can interfere in the reduction of vaccination coverage.Conclusion: The study points to the need for resignification of vaccination practices by professionals and users, with a view to disseminating truthful information and consolidating vaccination as a preventive measure for diseases.

DESCRIPTORS: Vaccination; Access to information; Immunization programs.

\section{RESUMEN}

Objetivo: comprender los aspectos teóricos y conceptuales relacionados con la vacunación y las implicaciones de las Fake News. Método: Es una revisión narrativa de la literatura, las búsquedas se realizaron en bases Biblioteca Electrónica Científica en Línea (SciELO), Literatura Latinoamericana y del Caribe en Ciencias de la Salud (Lilacs) y Google Scholar, con 11 artículos seleccionados para análisis. La búsqueda se llevó a cabo en abril y mayo de 2020. Resultados: La adherencia a la vacunación se considera una estrategia importante que busca ampliar la cobertura de vacunación. Hoy en día es posible afirmar que las redes sociales son el principal medio de difusión de Fake News sobre vacunación, y de ahí que pueda interferir en la reducción de la cobertura de vacunación. Conclusión: El estudio apunta a la necesidad de resignificación de las prácticas de vacunación por parte de profesionales y usuarios, con miras a difundir información veraz y consolidar la vacunación como medida preventiva de enfermedades. DESCRIPTORES: Vacunación; Acceso a la información; Programas de vacunación.

RECEBIDO EM: 30/09/2020 APROVADO EM: 04/11/2020

\section{Lucas Benício Pinto}

Acadêmico de Enfermagem no Centro Universitário Vale do Salgado - UNIVS.

ORCID: 0000-0002-6982-6308. 


\section{João Paulo Xavier Silva}

Enfermeiro. Doutorando em Cuidados Clínicos na Universidade Estadual do Ceará (UECE). Mestre em Enfermagem. Docente do curso Enfermagem do Centro Universitário Vale do Salgado - UNIVS.

ORCID: 0000-0003-3082-9373.

\section{Maria Luiza Santos Ferreira}

Acadêmica de Enfermagem na Universidade Regional do Cariri - URCA.

ORCID: 0000-0002-2737-5385.

\section{Kerma Márcia de Freitas}

Enfermeira. Doutoranda em Saúde Coletiva pela Universidade de Fortaleza - UNIFOR. Mestre em Saúde Coletiva. Coordenadora do curso de Enfermagem do Centro Universitário Vale do Salgado - UNIVS.

ORCID: 0000-0003-3021-4758.

\section{Roberta Peixoto Vieira}

Enfermeira. Mestra em Saúde da Criança e do Adolescente. Docente do curso Enfermagem do Centro Universitário Vale do Salgado - UNIVS.

ORCID: 0000-0003-0640-1772

\section{INTRODUÇÃO}

A imunização corresponde à utilização de um determinado imunobiológico, com o objetivo de estimular o sistema imunológico a criar defesas contra patógenos específicos que podem ser nocivos ao organismo. Nos serviços de saúde, a imunização é efetivada pela prática da vacinação ${ }^{1}$.

No Sistema Único de Saúde (SUS) brasileiro, instituído pela Constituição Federal, a vacinação se apresenta como uma das principais estratégias de proteger a população contra doenças imunopreveníveis. É reconhecida como ação prioritária e obrigatória, sendo realizada majoritariamente pela Estratégia Saúde da Família².

$O$ processo de vacinação ofertado gratuitamente pelo Sistema Único de Saúde no âmbito da Estratégia de Saúde da Família tem gerado grandes resultados na esfera de saúde pública, pois tanto sua efetividade como a questão do custo mostraram resultados positivos no cenário nacional. $\mathrm{Ou}$ seja, tem favorecido melhoras na qualidade de vida da população, no que diz respeito ao enfrentamento de doenças até então desconhecido, mas que na atualidade se conformam métodos eficazes de controle e prevenção dessas doenças ${ }^{3}$.

No Brasil, o Programa Nacional de Imunização é responsável pela supervisão, normatização e implantação de ações e políticas que concretizam o uso de imunobiológicos.
A Organização Mundial de Saúde (OMS) estabelece os parâmetros a serem seguidos e o Programa Nacional de Imunização (PNI) faz desses parâmetros metas a serem efetivadas com a finalidade de tornar as vacinas acessíveis em todo o território nacional ${ }^{4}$.

A Estratégia de Saúde da Família, como o principal local para a realização da vacinação, se configura neste cenário como a porta de entrada do sistema de saúde, tendo a equipe de enfermagem como responsável pela execução desse procedimento, com vistas à consolidação dos benefícios no âmbito da Atenção Primária à Saúde. Desta forma, o exercício do trabalho na Atenção Primária a Saúde requer desses profissionais conhecimentos cabíveis a essa prática e um planejamento de ações que busquem compreender as condiçốes sociais dos usuários, a questão geográfica para o acesso, as orientações e informações referentes à vacinação, entre outras medidas para favorecer o vínculo profissional-usuário 5 .

Contudo, na contemporaneidade, o uso disseminado das redes sociais tem favorecido cada vez mais a expansão de informações falsas, as Fake News, e esse contexto tem contribuído negativamente nas práticas de vacinação. Devido à popularização da internet e consequente aumento no tráfego de informações nas mídias mais comuns, possibilitou-se um meio para a criação e divulgação de dados muitas vezes falsos sobre assuntos de extrema importância como a vacinação ${ }^{6}$.
Destarde, ao implicar-se no âmbito da adesão às práticas preventivas de saúde, as Fake News também podem favorecer o aumento gradativo do número de pessoas não vacinadas no país, contribuindo para o reaparecimento de doenças imunopreveníveis e o comprometimento da saúde da população. Desta maneira, um processo educativo e de promoção da consciência sanitária deve ser executado pelos profissionais de saúde ${ }^{7}$.

Esse artigo tem como objetivo compreender por meio de uma revisão narrativa da literatura os aspectos teórico-conceituais relacionados à vacinação e as implicações das Fake news. Justifica-se pela necessidade de investigar, por meio da literatura científica, as relações entre a vacinação e as Fake News. Torna-se relevante pelo fato de que se vivencia na atualidade uma onda de movimentos anti vacina, advindos a disseminação de informações não verídicas sobre os imunobiológicos.

\section{MÉTODO}

Trata-se de uma revisão da literatura, do tipo narrativa. As revisões narrativas possibilitam a descrição e discussão de um objeto de estudo considerando o campo teórico de produção de conhecimento. Desse modo, realiza-se uma análise da literatura científica convergindo em uma interpretação e análise crítica pessoal do autor ${ }^{8}$.

Delinearam-se como perguntas nortea- 
doras: Quais os aspectos teórico-conceituais relacionados à vacinação e às Fake news? Quais são as implicaçôes das Fake News no processo de vacinação? O material textual utilizado foi selecionado por meio de buscas parametrizadas, elegendo-se bases de dados de acesso livre como Scientific Electronic Library Online (SciELO), Literatura Latino-americana e do Caribe em Ciências da Saúde (Lilacs) e Google Scholar. O período da coleta de dados se deu entre abril e maio de 2020.

Aplicaram-se os descritores em ciências da saúde (DeCS) "Vacinação", "Acesso a informação" e "programas de imunização" utilizando-se "AND" como operador booleano. A elegibilidade do material se deu por meio dos critérios de inclusão: textos completo do tipo artigos, sem recorte temporal e idioma português, inglês e espanhol; e critérios de exclusão: estudos de revisão e estudos que não abordassem a temática. A seleção se deu com aplicação dos critérios de elegibilidade e, sequencialmente, leitura de títulos, resumos e, quando elegíveis, leitura completa dos artigos. A análise e interpretação dos artigos selecionados se deu pela sumarização dos achados e pela técnica da categorização temática, possibilitando a apresentação dos resultados.

\section{RESULTADOS}

A busca eletrônica resultou na identifi- cação inicial de 72 artigos que, após triagem, resultou em um total de 11 artigos selecionados para a realização desta revisão. Dentre esses, houve uma maior concentração referente à produção temática entre os anos 2018 e 2019, o que reafirma a contemporaneidade dessa temática.

$\mathrm{O}$ quadro abaixo apresenta a distribuição das produções identificadas pela identificação do ano, título e periódico de publicação.

O processo analítico-interpretativo dos achados dessa revisão possibilitou a delineação de duas categorias temáticas que serão apresentadas a seguir, foram elas: 1 . Vacinação: aspectos conceituais e execução na Atenção Primária à Saúde; e 2. Fake news e suas implicaçỗes na vacinação.

Quadro 1 - Estudos elegíveis mediante buscas nas bases SciELO, Lilacs e Google Scholar.

\begin{tabular}{|c|c|c|}
\hline ANO & TÍTULO DO ARTIGO & PERIÓDICO \\
\hline 2015 & $\begin{array}{l}\text { A Internet como interface na relação médico- } \\
\text {-paciente: aliada ou inimiga? }\end{array}$ & Revista médica de Minas Gerais \\
\hline 2015 & $\begin{array}{c}\text { Fatores associados à adesão à } \\
\text { vacinação anti-influenza em idosos não } \\
\text { institucionalizados }\end{array}$ & Cadernos de Saúde Pública \\
\hline 2016 & $\begin{array}{l}\text { Educação para o trabalho em sala de vacina: } \\
\text { percepção dos profissionais de enfermagem }\end{array}$ & $\begin{array}{l}\text { Revista de Enfermagem do } \\
\text { Centro Oeste Mineiro }\end{array}$ \\
\hline 2017 & $\begin{array}{l}\text { Análise da ocorrência de evento adverso pós- } \\
\text {-vacinação decorrente de erro de imunização. }\end{array}$ & $\begin{array}{l}\text { Revista Brasileira de Enferma- } \\
\text { gem }\end{array}$ \\
\hline 2017 & $\begin{array}{c}\text { Avaliação da organização e funcionamento } \\
\text { das salas de vacina na atenção primária à } \\
\text { saúde }\end{array}$ & $\begin{array}{l}\text { Revista Epidemiologia e Servi- } \\
\text { ços de Saúde }\end{array}$ \\
\hline 2018 & $\begin{array}{l}\text { Pós-verdade e fontes de informação: um } \\
\text { estudo sobre fake news }\end{array}$ & Revista Conhecimento em Ação \\
\hline 2018 & $\begin{array}{c}\text { Educação permanente em sala de vacina: } \\
\text { qual a realidade? }\end{array}$ & $\begin{array}{l}\text { Revista Brasileira de Enferma- } \\
\text { gem }\end{array}$ \\
\hline 2018 & $\begin{array}{l}\text { A dupla epidemia: febre amarela e desinfor- } \\
\text { mação }\end{array}$ & $\begin{array}{l}\text { Revista Eletrônica de Comuni- } \\
\text { cação Informação e Inovação } \\
\text { em Saúde }\end{array}$ \\
\hline 2019 & $\begin{array}{l}\text { Saúde sem fake news: estudo e caracteri- } \\
\text { zação das informações falsas divulgadas } \\
\text { no canal de informação e checagem de fake } \\
\text { news do ministério da saúde. }\end{array}$ & Revista Mídia e Cotidiano \\
\hline 2019 & $\begin{array}{c}\text { A percepção da equipe de enfermagem } \\
\text { sobre a segurança do paciente em sala de } \\
\text { vacinação }\end{array}$ & Revista Cuidarte \\
\hline 2019 & Imunização na atenção básica & $\begin{array}{l}\text { Revista multidisciplinar de } \\
\text { pesquisa }\end{array}$ \\
\hline \multicolumn{3}{|c|}{ Fonte: Dados da pesquisa, 2020.} \\
\hline
\end{tabular}

\section{DISCUSSÃO}

\section{Vacinação: aspectos conceituais e execução na Atenção Primária à Saúde}

A vacina é umas das maiores descobertas da humanidade, tornando-se uma realidade através das pesquisas pelo médico Edward Jenner após 20 anos de estudos, em 1976. A etiologia da palavra vacina deriva-se dos termos vaccine e vaccination, que são derivados do termo latino vacca, com o qual a vacinação guarda importante relação. Constitui-se de um composto que em sua formulação apresenta patógenos específicos de forma atenuada. Este produto faz com que o indivíduo vacinado produza defesas corporais que entram em ação quando houver de fato contato com o patógeno ${ }^{1}$.

Operacionalmente, processo de vacinação está contido fundamentalmente nos serviços ofertados pela Atenção Primária à Saúde, que é um modelo de nalmente a partir de 1978, no qual a declaração de Alma-Ata estabeleceu a Atenção Primária à Saúde como medida que garante a sustentação dos serviços de saúde com a oferta de serviços à população de maneira regional, integra, contínua e sistematizada. No contexto brasileiro, pode-se afirmar que a consolidação da Atenção Primária à Saúde se deu com a atenção à saúde consolidado internacio- 
criação e implantação, no ano de 1994, do Programa Saúde da Família, hoje denominado Estratégia Saúde da Família?

A Estratégia de saúde da Família conta com atividades desenvolvidas por uma equipe multidisciplinar, e possui como um de seus objetivos centrais e mais relevantes a reorganização do modo como estes serviços são prestados aos usuários. Dentre estes muitos serviços, a vacinação encontra terreno fértil nesse cenário, pois é uma ação de caráter rotineiro e contínuo e que marca grandes avanços na saúde nas últimas décadas, consequentemente acaba por exercer grande influência nas condições de saúde da população ${ }^{10}$.

Quando o assunto se refere à adesão ao processo de vacinação e como consequência disto a imunização, é essencial e de grande valor expor sobre as vantagens, privilégios $\mathrm{e}$ a resultância desta escolha. A adesão à vacinação é observada como uma importante estratégia que busca ampliar cada vez mais a cobertura vacinal propostas nas campanhas e nos serviços de rotina da atenção básica, contribuindo para firmar proteção à população. Nesse contexto, envolve também o conhecimento e as informações que a população recebe acerca do assunto ${ }^{11}$.

\section{Fake news e suas implicações na vacinação}

O termo Fake News é definido como um conjunto de informações que representam uma situação ou ponto de visto a respeito de um determinado acontecimento, mas que contém em seu corpo de dados preposições falsas. Seu conteúdo é desenvolvido com a finalidade de enganar os leitores que tenham acesso a seu material, com isso gerando a difusão de inverdades ${ }^{12}$.

As Fake News tornaram-se reconhecidas de maneira mais popular após o término da campanha presidencial dos Estados Unidos entre Donald Trump e Hilary Clinton, onde os eleitores criavam e difundiam inverdades sobre os candidatos de oposição nas mídias sociais. Na contemporaneidade, o uso da internet como ferramenta da era moderna, advinda da evolução da tecnologia e dos avanços da humanidade, está presente no dia a dia em inúmeros contextos da sociedade, inclusive no setor saúde ${ }^{13,6}$.

É fato que nas redes sociais a criação e propagação de notícias falsas fortalecem grupos que contestam as práticas habituais no âmbito da saúde, como os movimentos antivacinas. Contudo, vale ressaltar que medidas devem ser tomadas visando combatê-los. Devem-se constituir espaços para divulgação de notícias verídicas e com embasamento científico comprovado e sua consequente disseminação, auxiliando no esclarecimento de questões e contribuindo de maneira efetiva no combate a inverdades, que podem trazer malefícios a toda sociedade ${ }^{14}$.

No ambiente virtual, a propagação de notícias falsas em uma escala mundial direciona para um fator preocupante, a diminuição da importância da verdade. Desde o ano de 2016 que por meio das redes sociais, o termo Fake News ganhou popularidade e viralizou constantemente nas interações sociais, direcionando-se para diversos setores na vida das pessoas, tanto em termos políticos como também na saúde pública. Neste ponto, o campo da vacinação sofre diversas interferências e ataques com publicações e disseminações de notícias infundadas, gerando na população incertezas e inseguranças acerca da adesão a este processo ${ }^{6}$.

$\mathrm{Na}$ atualidade, é possível afirmar que as mídias sociais são os principais meios de propagação das Fake News sobre vacinação, e a partir disto pode interferir na diminuição da cobertura das campanhas de vacinação propostas pelo Programa $\mathrm{Na}$ cional de Imunização. Infere-se que através do uso irresponsável de plataformas virtuais como Facebook, Twitter, WhatsApp e navegadores de internet, a disseminação por meio de vídeos, notícias ou ainda portais de comunicação criados para esses fins, ganham proporções inimagináveis na população e expressam informações contrárias ao real benefício das vacinas ${ }^{15}$.

Desse modo, um dos principais alvos de ataque das Fake News é a saúde pública, de maneira mais específica as campanhas de vacinação, e sua propagação ganha mais força pelo fato de que os assuntos que estas abordam estão relacionados a aspectos do cotidiano. Desta maneira, estes informes ganham maior especulação quando estão sendo disseminados durante pandemias ou doenças graves, esta junção entre inverdade e acontecimentos reais na sociedade, juntamente uma menor compreensão por grande parte das pessoas acerca desses assuntos favorece e torna as Fake News perigosas e prejudiciais ao bem estar social ${ }^{7,14}$.

De acordo com estudos anteriores que realizaram buscas nas plataformas virtuais, foram encontradas mais de 400 Fake News relacionadas às vacinas, expressando assuntos como: vacinas podem causar autismo; as vacinas apresentam efeitos colaterais que são prejudiciais à saúde, tanto a curto como longo prazo; vacinas conjugadas entre a poliomielite, difteria, tétano e coqueluche podem ocasionar morte súbita nas crianças; vacinas contemplam mercúrio em sua composição; realizar a administração de mais de um imunobiológicos em uma criança pode causar efeitos colaterais severos prejudiciais à mesma; o processo de vacinação pode ser até fatal $1^{9,12}$.

No período entre os anos de 2013 e 2015 foram notificados mais de mil casos de sarampo nos estados do Ceará e Pernambuco, e até o ano de 2019 foram notificados no Brasil 92 casos da doença espalhados pelos estados de Amazonas, Roraima, Pará, São Paulo, Santa Catarina, Rio de Janeiro e Minas Gerais. Uma das prováveis razões para estes acontecimentos é a formulação e propagação de boatos e mitos acerca das vacinas, mas não somente isto contribui, pois a falta de imunobiológicos, a percepção das pessoas de que as doenças desapareceram e que não se necessita mais fazer uso das vacinas, juntamente com a carência do conhecimento do calendário vacinal resultam em uma diminuição da adesão populacional nas campanhas causando assim redução das coberturas vacinais à níveis preocupantes ${ }^{16}$.

Diante disto, nota-se a importância da implantação de estratégias que possibilitem a formulação do desenvolvimento crítico de profissionais e da população à respeito de assuntos voltados ao âmbito da saúde. Para que isto ocorra, é necessário ressaltar a importância dos profissionais de saúde, principalmente os que atuam 
na Atenção Primária a Saúde, por meio da Estratégia de Saúde da Família que atuam com base na análise das condiçôes de saúde da população para a prestação de serviços adequados que visam a garantia da qualidade de vida e prevenção de doenças dos usuários ${ }^{17,18}$.

Portanto, torna-se fundamental que os profissionais de saúde possam manter um diálogo contínuo com a população a fim de apurar as notícias relacionadas à saúde e assim conscientizá-los sobre o esclarecimento dos boatos e mentiras que estão presentes na comunidade por intermédio das mídias sociais. Desta forma, a realização de boas práticas pelos profissionais de saúde, mais específico os que atuam na APS, na divulgação de informações de saúde de fontes seguras e com embasamento cientifico, são responsáveis por quebrar tabus, estigmas, desmistificar inverdades e disseminar a boa informação. Isso faz com que o usuário tome conhecimento acerca do tema e permita que ele escolha de maneira segura, consciente e responsável as decisões a serem tomadas e as notícias que devem ser compartilhadas ou não ${ }^{19}$.

\section{CONCLUSÃO}

A presente revisão contribuiu para uma maior compreensão acerca dos aspectos teórico-conceituais relacionados à vacinação e as implicações das Fake news nesse processo. Elucidou questões fundamentais relacionadas à vacinação e sua importância, elencando de que modo à disseminação de informações falsas podem interferir negativamente na adesão dos usuários.

A literatura acena para a necessidade da ressignificação das práticas de vacinação por parte dos profissionais e usuários do serviço, com vistas a consolidar essa estratégia como medida preventiva de doenças. Salienta-se a necessidade de investigar essa temática também no campo prático, com vistas à melhor compreensão desse fenômeno e sua ocorrência no meio social. -

\section{REFERÊNCIAS}

1. Ballalai I, Bravo F. Imunização: tudo o que você sempre quis saber. Rio de Janeiro: RMCOM, 2016.

2. Araújo TM, Souza FO, Pinho PS. Vacinação e fatores associados entre trabalhadores de saúde. Cadernos de Saúde Pública. Rio de Janeiro. 2019;35(04):02-14.

3. Brasil. Ministério da Saúde. Secretaria de Vigilância em Saúde. Departamento de Vigilância das Doenças Transmissíveis. Manual de Normas e Procedimentos para Vacinação. Brasília: Ministério da Saúde, 2014.

4. Barros MGM, Santos MCS, Bertolini RPT, Netto VBP, Andrade MS. Perda de oportunidade de vacinação: aspectos relacionados à atuação da atenção primária em Recife. Epidemiol. Serviços de Saúde. Brasília. 2015;24(04):701-710.

5. Montserrat CJ, Godoy P, Marsal JR, Barbé IF. Factores asociados a recibir la vacinación antigripal em profesionales de atención primaria. Gac Sanit. Barcelona. 2015;29(05):383-386.

6. Saraiva LJC, De Faria JF. Ciência e a Mídia: a propagação de Fake News e sua relação com o movimento anto-vacina no Brasil. Sociedade Brasileira de Estudos Interdisciplinares da Comunicação. Pará. 2019;42(01):01-15.

7. Henriques CMP. A dupla epidemia: febre amarela e desinformação. Revista Eletrônica de Comunicação Informação e Inovação em Saúde. Brasília. 2018;12(01):09-13.

8. Rother, ET. Revisão sistemática X Revisão narrativa. Acta Paulista Enfermagem. São Paulo. 2007. 20(2):5-6.

9. Zinelli AGV, Marcelino DM, Tibola ES, Gôes FC, Silva RF, Melo FAO, Silva F. Imunização na atenção básica. Revista Multidisciplinar e de Pesquisa. Mato Grosso do Sul. 2019;13(47):499-507.

10. Siqueira LC, Martins AMEBL, Versiani CMC, Almeida LAV, OIiveira, CS, Nascimento JE, et al. Avaliação da organização e funcionamento das salas de vacina na atenção primária à saúde em Montes Claros, Minas Gerais. Revista Epidemiol. Serv. Saúde. Brasília. 2017;26(3):557-568.
11. Moura RF, Andrade FB, Duarte YAO, Lebrão ML, Antunes JLF. Fatores associados à adesão à vacinação anti-influenza em idosos não institucionalizados. Caderno de Saúde Pública. Rio de Janeiro. 2015;31(1):2157-2168.

12. Paula LT, Blanco YA, Silva TRS. Pós-verdade e fontes de informação: um estudo sobre fake news. Revista Conhecimento em Ação. Rio de Janeiro, 2018;2(1):93-110.

13. CASTRO E. A Internet como interface na relação médico-paciente: aliada ou inimiga? Revista CRM-PR. Paraná. 2015;32(126):1-12.

14. Monari ACP, Bertolino Filho C. Saúde sem fake news: estudo e caracterização das informações falsas divulgadas no canal de informação e checagem de fake news do ministério da saúde. Revista Mídia e Cotidiano. Rio de Janeiro. 2019;13(1):160-186.

15. Vieira LM; Silva NR, Cordeiro DF. Análise descritiva das fake news da saúde através de mineração de textos no Portal da Saúde. Congresso de Ciências da Comunicação na Região Centro-Oeste. 2019 Jun 1-4 Goiânia, GO.

16. Modelli L. Idoso pode se vacinar contra sarampo? Quem já teve deve tomar a vacina? Especialista respondem dúvidas. BBC News Brasil, 2018.

17. Oliveira VC, Rennó HMS, Santos YR, Rabelo AFG, Gallardo MDPS, Pinto IC. Educação para o trabalho em sala de vacina: percepção dos profissionais de enfermagem. Revista de Enfermagem do Centro Oeste Mineiro. Minas Gerais. 2016;6(3):23312341.

18. Oliveira VC, Tavares LOM, Maforte NTP, Silva LNLR, Rennó HMS, Amaral GG, et al. A percepção da equipe de enfermagem sobre a segurança do paciente em sala de vacinação. Revista Cuidarte. Colômbia. 2019;10(1): 1-12.

19. Martins JRT, Bruna GPA, Oliveira VCO, Viegas SMF. Educação permanente em sala de vacina: qual a realidade? Revista Brasileira de Enfermagem. Minas Gerais. 2018;71(1):15-724. 